\title{
The Effectiveness of Pulmonary Rehabilitation on Pulmonary Function among Adults Patients of COVID- 19 Survivors: A Systematic Review
}

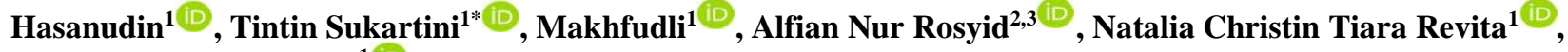 \\ Handira Nadhifatul Aini ${ }^{1}$ (D) \\ ${ }^{1}$ Faculty of Nursing, Universitas Airlangga, Surabaya, Indonesia. \\ ${ }^{2}$ Department of Pulmonology and Respiratory Medicine, Faculty of Medicine, Universitas Airlangga, Surabaya, Indonesia. \\ ${ }^{3}$ Universitas Airlangga Hospital, Surabaya, Indonesia.
}

\begin{tabular}{l} 
ARTICLE INFO \\
\hline Article history: \\
Received 25 October 2021 \\
Received in revised form 12 \\
January 2022 \\
Accepted 18 January 2022 \\
Available online 30 January 2022 \\
\hline Keywords: \\
Infection disease, \\
Long COVID, \\
Pulmonary function, \\
Pulmonary rehabilitation.
\end{tabular}

\section{INTRODUCTION}

Corona Virus Infection Disease (COVID-19) refers to the infectious disease caused by Severe Acute Respiratory Syndrome Coronavirus 2 (SARS-CoV-2). World Health Organization (WHO) has declared COVID-19 as a pandemic on 11 March $2020{ }^{1}$

Global data for COVID-19 as of 29 September 2021 showed as many as 232.6 million people confirmed

\begin{abstract}
Introduction: Corona Virus Infection Disease (COVID-19) refers to the infectious disease caused by Severe Acute Respiratory Syndrome Coronavirus 2 (SARS-CoV-2). Most of COVID-19 survivors have residual symptoms after being discharged from the hospital, it then leads to the decreased lung function and quality of life (QoL). Pulmonary rehabilitation is essential because it can reduce the impact of these sequelae. This study aimed to determine the effectiveness of pulmonary rehabilitation in improving lung function among adult patients of COVID-19 survivors.

Methods: This study was conducted using 5 databases, namely: CINAHL, PubMed, SAGE, Science Direct, and Scopus from 1 April 2020 to 30 September 2021. The combination of keywords used was: "Pulmonary Rehabilitation", "Pulmonary Function", and "Post-COVID-19". In assessing the bias and methodological quality of the articles obtained, this study used the Joanna Briggs Institute (JBI) critical appraisal checklist tools.

Results: From 678 articles, 11 research articles met the criteria. Pulmonary rehabilitation performed in post-COVID-19 patients included the provision of aerobic exercise, breathing exercises, strength training, balance exercises, relaxation, occupational therapy, psychological support, and nutrition consultation. Lung rehabilitation mainly was performed two sessions a week for 3-8 weeks with different proportions for each patient according to clinical conditions and ability limits. Spirometry and the 6-minute walk test (6MWT) revealed that patients who completed the pulmonary rehabilitation program experienced improvements in their lung function. Conclusion: Comprehensive pulmonary rehabilitation programs under supervision and psychotherapists are the right choice in improving lung function among COVID-19 survivor adult patients.
\end{abstract}

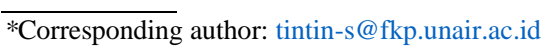

Jurnal Respirasi (Journal of Respirology), p-ISSN: 2407-0831; e-ISSN: 2621-8372.

Accredited No. 200/M/KPT/2020; Available at https://e-journal.unair.ac.id/JR. DOI: 10.20473/jr.v8-I.1.2022.15-25

(c) (i) (2) This work is licensed under a Creative Commons Attribution-Share Alike 4.0 International License. 
One of the causes of sequelae in post-COVID-19 patients is abnormalities in the lungs due to SARS-CoV2 infection. On follow-up computed tomography (CT) scan, $46(56.8 \%)$ of 81 patients in the study cohort did not have lung abnormalities, and $35(43.2 \%)$ patients had residual lung opacities. Ground glass opacity (GGO) $(16 / 35 ; 45.7 \%)$ was the most common residual abnormality than parenchymal bands $(9 / 35 ; 25.7 \%)$, a mixed pattern of GGO and parenchymal bands $(6 / 35$; $17.2 \%)$, bronchiectasis $(6 / 35 ; 17.2 \%)$, and interlobular septal thickening $(4 / 35 ; 11.4 \%)$. The characteristics of patients with lung abnormalities were old age, had a higher body mass index (BMI), had more comorbidities, had lower $\mathrm{SpO}_{2}$, were in the hospital longer, had a greater probability of intensive care unit (ICU) admission, had a higher white blood cell (WBC) count, and had a higher CT severity score. ${ }^{4}$

From 145 COVID-19 patients assessed 100 days after being discharged from the hospital, $41 \%$ of all subjects showed persistent symptoms with shortness of breath as the most frequently reported symptom. $21 \%$ of patients with COVID-19 had decreased diffusion capacity. ${ }^{5}$ The follow-up results from 1733 post-COVID patients six months after acute infection. Most of the patients had the main complaint of experiencing fatigue quickly during activities, muscle weakness, insomnia, anxiety, and even depression. ${ }^{6}$

Rehabilitation in post-COVID-19 patients is essential because it can reduce the impact of sequelae on patients, improve lung function, reduce dyspnea during activities, and reduce anxiety and depression to improve their quality of life (QoL). ${ }^{7}$ This study aimed to determine the effectiveness of pulmonary rehabilitation in improving lung function among adult patients of COVID-19 survivors.

\section{METHODS}

A review guide was prepared to guide compiling this study. These guidelines covered the rationale and objectives of the study, eligibility criteria for studies to be included in the article, sources of information, search strategies used, article selection and data collection process, results obtained, methods for assessing the risk of bias, and results from data synthesis. This study followed the preferred reporting item guidelines for systematic review and meta-analysis (PRISMA). ${ }^{8}$

\section{Search Strategy}

A systematic review was performed using 5 electronic databases, namely CINAHL, PubMed, SAGE, Science Direct, and Scopus. The search was conducted on 30 September 2021. These keywords were applied using Boolean Logic (and, or) in searching for the articles. The MeSH keyword and term combinations developed were then adapted to other databases. The keywords and subject titles used in searching the article were ("Pulmonary Rehabilitation AND "Pulmonary Function", AND "Post COVID-19") published from 1 April 2020 to 30 September 2021 in English.

\section{Inclusion and Exclusion Criteria}

The inclusion criteria used the population, intervention, comparison, outcome and time (PICOT) framework, where the population was post-COVID-19 patients (Table 1). The given intervention focused on pulmonary rehabilitation. The primary outcome of this study was an improvement in lung function among COVID-19 survivors. The articles taken were the last two years using the cohort study method and randomized controlled trials (RCT). Articles without full text and abstracts and articles that did not give detailed intervention, were excluded.

\section{Study Selection}

According to PRISMA guidelines, the first step was to search for articles which matched the topic in an electronic database. After getting the article, it was selected and then the same article was deleted After that, the title and abstract were filtered to meet the eligibility

Table 1. Picot framework

\begin{tabular}{ll}
\hline PICOT Framework & Inclusion and Exclusion Criteria \\
\hline Population & Post-COVID-19 patients \\
Intervention & Pulmonary rehabilitation \\
Comparison & No comparison intervention \\
Outcomes & Pulmonary function \\
Time & 1 April 2020 - 30 September 2021 \\
Study Design & Cohort Study and RCT \\
Language & English \\
& Articles that did not discuss pulmonary rehabilitation in post-COVID-19 patients, articles \\
Exclusion criteria: & without full text and abstracts without intervention details, dated before 2020, and studies \\
& conducted in languages other than English, as well as non-cohort Study and RCT designs were \\
& excluded. \\
\hline
\end{tabular}


criteria. The whole text of each selected article which met the inclusion criteria was taken for further examination. A secondary search was performed from the article reference list to identify additional notes. The final stage included relevant articles and met all inclusion criteria in a systematic review. Three independent reviewers carried out the search and screening process.

\section{Risk of Bias}

The Joanna Brings Institute (JBI) critical appraisal checklist was used to assess the methodological quality of the articles included. ${ }^{9}$ Three independent reviewers assessed the risk of bias for each included study. Any disagreements were resolved by discussion.

\section{Data extraction}

A structured form was used to extract information from the included articles starting from the author, year, country, method, sample, age, duration and frequency, intervention, outcome, and the results of the articles to evaluate the effect of the intervention. These data are summarized in Table 2.

\section{RESULTS}

\section{Study selection}

A total of 678 articles were identified, the duplicates were removed, and the remaining 673 articles were taken for a feasibility review. The articles were reviewed according to the abstracts of the remaining 197 articles. From the full-text article feasibility test, there were 54 articles left, and 11 research articles were taken for review (Figure 1).

\section{Risk of Bias}

Figure 2 shows the overall risk assessment of bias. Almost all of the studies that had been analyzed for risk of bias using JBI with RCT methodologies and cohort studies had a low risk of bias. It was difficult to blind the patient due to the availability of pulmonary rehabilitation therapies. One article did not explain how long pulmonary rehabilitation was offered on regularly.

The study was identified through database searching, CINAHL (1), PubMed (78),

SAGE (87), Science Direct (477), and Scopus (35) $(\mathrm{n}=678)$

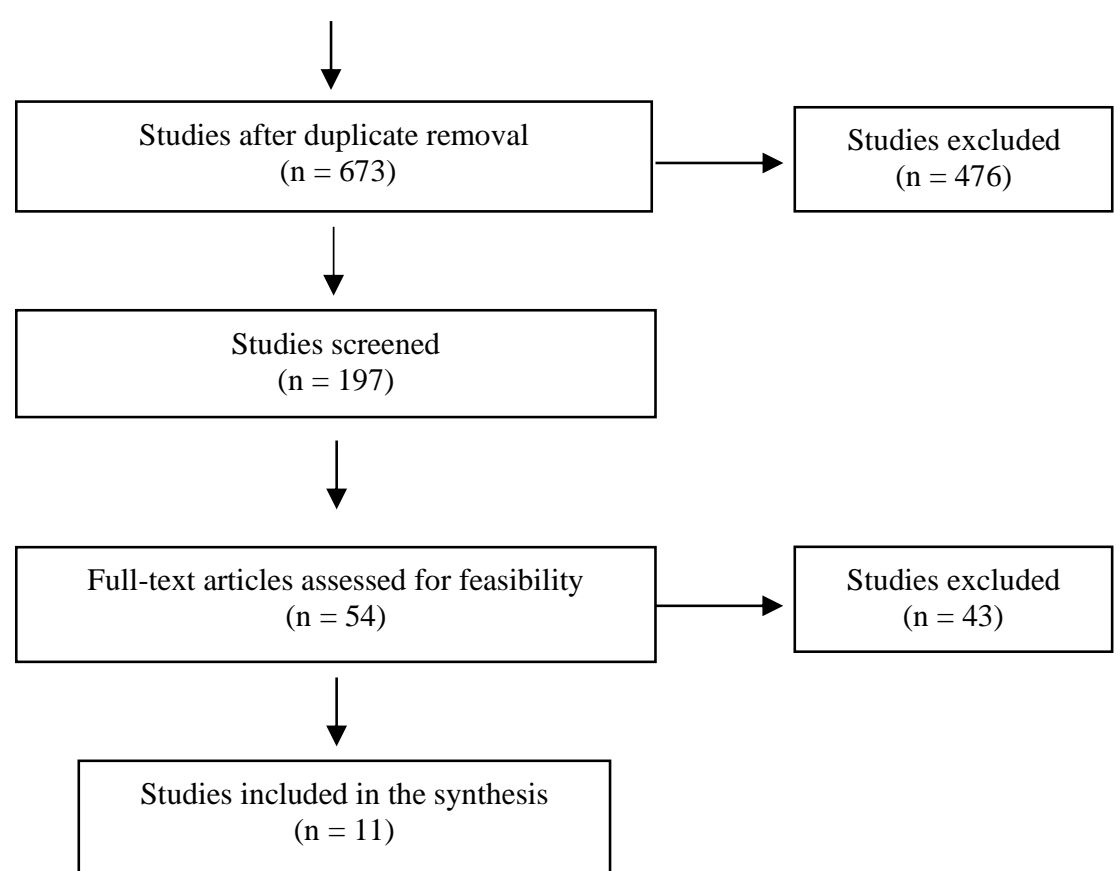

Figure 1. Flow chart of study selection 
Table 2. Description of the selected studies that used intervention pulmonary rehabilitation to increase pulmonary function among adult patients of COVID-19 survivors

\begin{tabular}{|c|c|c|c|c|c|c|c|c|c|}
\hline No. & Authors & Country & Methods & Samples & $\begin{array}{c}\text { Average } \\
\text { Age }\end{array}$ & $\begin{array}{c}\text { Duration and } \\
\text { Frequency }\end{array}$ & Interventions & Outcomes & Results \\
\hline 1. & $\begin{array}{l}\text { Gloeckl, et } \\
\text { al., } 2021\end{array}$ & Germany & $\begin{array}{l}\text { Prospective } \\
\text { oservational } \\
\text { cohort study }\end{array}$ & $\begin{array}{l}50 \text { patients } \\
\text { (22 males, } \\
28 \text { females) }\end{array}$ & $\begin{array}{l}\text { The mean } \\
\text { age was } 52 \\
\text { years old } \\
\text { in } \\
\text { moderate } \\
\text { patients } \\
\text { and } 66 \\
\text { years old } \\
\text { in severe } \\
\text { patients }\end{array}$ & $\begin{array}{l}2-5 \text { sessions } \\
\text { per day for } 3 \\
\text { weeks }\end{array}$ & $\begin{array}{l}\text { 1. Endurance training, (10-20 minutes for } \\
5 \text { days per week) } \\
\text { 2. Strength training, (10-20 minutes for } 5 \\
\text { days per week) } \\
\text { 3. Respiratory physiotherapy ( } 30 \text { minutes } \\
\text { for } 4 \text { days per week) } \\
\text { 4. Activities of daily living training ( } 30 \\
\text { minutes for } 5 \text { days per week) } \\
\text { 5. Relaxation ( } 30 \text { minutes for } 2 \text { days per } \\
\text { week) } \\
\text { 6. Occupational therapy and psychological } \\
\text { support } \\
\text { 7. Psychological support } \\
\text { 8. Nutritional counselling }\end{array}$ & $\begin{array}{ll}\text { 1. } & \text { 6MWD } \\
\text { 2. } & \text { Lung function } \\
\text { 3. } & \text { FVC } \\
\text { 4. } & \text { QoL }\end{array}$ & $\begin{array}{l}\text { In post-COVID-19 patients, } \\
\text { pulmonary rehabilitation } \\
\text { proved a safe and effective } \\
\text { therapy alternative. }\end{array}$ \\
\hline 2. & $\begin{array}{l}\text { Sun, et al., } \\
2021\end{array}$ & China & $\begin{array}{l}\text { Prospective } \\
\text { clinical trial }\end{array}$ & $\begin{array}{l}31 \text { patients } \\
\text { (19 males, } \\
12 \text { females })\end{array}$ & $\begin{array}{c}60 \\
\text { years old }\end{array}$ & $\begin{array}{l}2 \text { sessions per } \\
\text { day for } 3 \\
\text { weeks }\end{array}$ & $\begin{array}{l}\text { Rehabilitation through tele-rehabilitation } \\
\text { included: } \\
\text { 1. Breathing exercises } \\
\text { 2. Respiratory muscle exercise ( } 15 \\
\text { minutes } 2 \text { times a day) } \\
\text { 3. Stretching training ( } 2 \text { times a day) } \\
\text { 4. Psychotherapy (listening to music for } \\
20 \text { minutes per day) }\end{array}$ & $\begin{array}{ll}\text { 1. } & \text { Exercise } \\
\text { endurance } \\
\text { 2. ADL } \\
\text { 3. } \\
\text { Blood oxygen } \\
\text { saturation } \\
\text { 4. Oxygen } \\
\text { intake } \\
\text { 5. Dyspnea }\end{array}$ & $\begin{array}{l}\text { After } 3 \text { weeks of pulmonary } \\
\text { rehabilitation and the } \\
\text { provision of medical therapy, } \\
\text { pulmonary rehabilitation } \\
\text { relieved symptoms of cough } \\
\text { and dyspnea in post-COVID- } \\
19 \text { patients. }\end{array}$ \\
\hline 3. & $\begin{array}{l}\mathrm{Al} \\
\text { Chikhanie, } \\
\text { et al., } 2021\end{array}$ & France & $\begin{array}{l}\text { Prospective } \\
\text { cohort study }\end{array}$ & $\begin{array}{l}21 \text { patients } \\
\text { (14 males, } \\
7 \text { females) }\end{array}$ & $\begin{array}{c}70 \\
\text { years old }\end{array}$ & $\begin{array}{l}1 \text { session } \\
\text { every week for } \\
6 \text { weeks }\end{array}$ & $\begin{array}{l}\text { Pulmonary rehabilitation consisted of: } \\
\text { 1. Breathing exercises } \\
\text { 2. Muscle strengthening and balance } \\
\text { 3. } 6 \text { minutes walk } \\
\text { 4. Cycling and exercise }\end{array}$ & $\begin{array}{l}\text { 1. PFT } \\
\text { 2. 6MWD } \\
\text { 3. QoL } \\
\text { 4. HADS }\end{array}$ & $\begin{array}{l}\text { Pulmonary rehabilitation was } \\
\text { able to improve the function } \\
\text { of post-ICU COVID-19 } \\
\text { patients but did not change } \\
\text { physical and psychosocial } \\
\text { disorders. }\end{array}$ \\
\hline 4. & $\begin{array}{l}\text { Spielmanns, } \\
\text { et al., } 2021\end{array}$ & Switzerland & $\begin{array}{l}\text { Prospective } \\
\text { design }\end{array}$ & $\begin{array}{l}99 \text { patients } \\
\text { (57 males, } \\
42 \text { females) }\end{array}$ & $\begin{array}{c}68 \\
\text { years old }\end{array}$ & $\begin{array}{l}\text { Maximum } 4 \\
\text { sessions per } \\
\text { day for } 3 \\
\text { weeks }\end{array}$ & $\begin{array}{l}\text { The pulmonary rehabilitation program } \\
\text { consisted of: } \\
\text { 1. Resistance training (10-30 minutes, 5-6 } \\
\text { times per week) } \\
\text { 2. Gymnastics ( } 45 \text { minutes, } 5-6 \text { times per } \\
\text { week) } \\
\text { 3. Take a walk outside ( } 45 \text { minutes, } 3-4 \\
\text { times per week) } \\
\text { 4. Strength training ( } 30 \text { minutes, twice or } \\
\text { thrice a week) } \\
\text { 5. Relaxation ( } 45 \text { minutes, } 2 \text { times per } \\
\text { week) } \\
\text { 6. Respiratory therapy ( } 30 \text { minutes, } 3 \\
\text { times per week) }\end{array}$ & $\begin{array}{ll}\text { 1. } & \text { HRQoL } \\
\text { 2. } & \text { FIM } \\
\text { 3. } & \text { HADS } \\
\text { 4. } & \text { CIRS } \\
\text { 5. } & \text { 6MWT } \\
\text { 6. } & \text { FT } \\
\text { 7. } & \text { PFT and } \\
& \text { blood gas } \\
& \text { analysis }\end{array}$ & $\begin{array}{l}\text { Pulmonary rehabilitation } \\
\text { programs were able to } \\
\text { improve clinical and } \\
\text { functional function in post- } \\
\text { COVID-19 patients, both } \\
\text { physical performances and } \\
\text { patient's well-being were } \\
\text { measured. }\end{array}$ \\
\hline
\end{tabular}




\begin{tabular}{|c|c|c|c|c|c|c|c|c|c|}
\hline 5. & $\begin{array}{l}\text { Daynes, et } \\
\text { al., } 2021\end{array}$ & $\begin{array}{c}\text { United } \\
\text { Kingdom }\end{array}$ & Cohort study & $\begin{array}{l}30 \text { patients } \\
(16 \text { males, } \\
14 \text { females })\end{array}$ & & $\begin{array}{l}2 \text { sessions per } \\
\text { week and } \\
\text { conducted for } \\
6 \text { weeks }\end{array}$ & $\begin{array}{l}\text { Pulmonary rehabilitation consisted of: } \\
\text { 1. Aerobic exercise (walking/treadmill) } \\
\text { 2. Strength training (lower and upper } \\
\text { limbs) }\end{array}$ & $\begin{array}{l}\text { 1. The } \\
\text { incremental and } \\
\text { endurance } \\
\text { shuttle walking } \\
\text { test (ISWT) } \\
\text { 2. CAT } \\
\text { 3. FACIT } \\
\text { 4. HADS } \\
\text { 5. EQ5D } \\
\text { 6. MoCA }\end{array}$ & $\begin{array}{l}\text { The pulmonary rehabilitation } \\
\text { program was safe for post- } \\
\text { COVID-19 patients and } \\
\text { showed an increase in } \\
\text { exercise capacity, reduced } \\
\text { symptoms of shortness of } \\
\text { breath, reduced and helped } \\
\text { overcome complaints of } \\
\text { fatigue. }\end{array}$ \\
\hline 6. & $\begin{array}{l}\mathrm{Li}, \text { et al., } \\
2021\end{array}$ & China & $\begin{array}{l}\text { Randomized } \\
\text { controlled trial }\end{array}$ & $\begin{array}{l}119 \text { patients } \\
(53 \text { males, } \\
66 \text { females })\end{array}$ & $\begin{array}{c}51 \\
\text { years old }\end{array}$ & $\begin{array}{l}\text { 3-4 sessions } \\
\text { every week } \\
\text { and conducted } \\
\text { for } 6 \text { weeks }\end{array}$ & $\begin{array}{l}\text { Pulmonary rehabilitation consisted of: } \\
\text { 1. Breathing control and thoracic } \\
\text { expansion } \\
\text { 2. Aerobic exercise } \\
\text { 3. Lower limb muscle strength exercises } \\
\text { specified in a three-tiered exercise plan } \\
\text { with difficulty and intensity scheduled } \\
\text { to increase over time }\end{array}$ & $\begin{array}{l}\text { 1. 6MWD } \\
\text { 2. Squat time in } \\
\text { seconds } \\
\text { 3. Pulmonary } \\
\text { function } \\
\text { 4. HRQoL } \\
\text { 5. Dyspnoea }\end{array}$ & $\begin{array}{l}\text { The TERECO program } \\
\text { provided had good } \\
\text { effectiveness in post-COVID- } \\
19 \text { patients. The best score } \\
\text { was found in improving QoL. }\end{array}$ \\
\hline 7. & $\begin{array}{l}\text { Büsching G, } \\
\text { et al., } 2021\end{array}$ & Switzerland & $\begin{array}{l}\text { Retrospectively } \\
\text { cohort study }\end{array}$ & $\begin{array}{l}51 \text { patients } \\
\text { (38 males, } \\
23 \text { females) }\end{array}$ & $\begin{array}{c}38 \\
\text { years old }\end{array}$ & $\begin{array}{l}\text { Rehabilitation } \\
\text { was given for } \\
3 \text { weeks in } \\
\text { post-COVID- } \\
19 \text { patients }\end{array}$ & $\begin{array}{l}\text { Pulmonary rehabilitation consisted of: } \\
\text { 1. Physiotherapeutic units consisted of } \\
\text { cardiopulmonary training (cycling, } \\
\text { guided walking) } \\
\text { 2. Strength exercise (weight training on } \\
\text { machines, free weight, elastic } \\
\text { resistance bands) } \\
\text { 3. Breathing exercises (deep breathing, } \\
\text { sputum evacuation), relaxation } \\
\text { techniques (progressive muscle } \\
\text { relaxation) } \\
\text { 4. Nutritional } \\
\text { 5. Occupational therapy and social } \\
\text { services. }\end{array}$ & $\begin{array}{l}\text { 1. } 6 \mathrm{MWT} \\
\text { 2. CRQ } \\
\text { 3. FIM }\end{array}$ & $\begin{array}{l}\text { Regardless of the severity of } \\
\text { illness, pulmonary } \\
\text { rehabilitation might enhance } \\
\text { physical performance and } \\
\text { QoL in post-COVID-19 } \\
\text { patients. }\end{array}$ \\
\hline 8. & $\begin{array}{l}\text { Stavrou, et } \\
\text { al., } 2021\end{array}$ & Greece & Cohort study & $\begin{array}{l}20 \text { patients } \\
(15 \text { males, } \\
5 \text { females })\end{array}$ & $\begin{array}{c}38 \\
\text { years old }\end{array}$ & $\begin{array}{l}3 \text { sessions per } \\
\text { week and } \\
\text { conducted for } \\
8 \text { weeks }\end{array}$ & $\begin{array}{l}\text { Pulmonary rehabilitation consisted of: } \\
\text { 1. Warm-up ( } 5 \text { minutes) } \\
\text { 2. Recovery set ( } 5 \text { minutes) with } \\
\text { flexibility and mobility exercises } \\
\text { 3. The aerobic exercise set with walking } \\
\text { ( } 50 \text { minutes) } \\
\text { 4. The set with yoga exercises to improve } \\
\text { breathing ( } 20 \text { minutes) } \\
\text { 5. The set with multi-joint strength } \\
\text { exercises ( } 20 \text { minutes) }\end{array}$ & $\begin{array}{l}\text { 1. Anthropometric } \\
\text { characteristics } \\
\text { 2. PFT } \\
\text { 3. Oxidative stress } \\
\text { biomarkers } \\
\text { 4. 6MWT }\end{array}$ & $\begin{array}{l}\text { A pulmonary rehabilitation } \\
\text { program can be a beneficial } \\
\text { and effective practice in the } \\
\text { recovery of symptoms in } \\
\text { post-COVID-19 patients as } \\
\text { well as an innovative strategy } \\
\text { for the treatment of sequelae } \\
\text { SARS-CoV-2 infection- } \\
\text { related fatigue. }\end{array}$ \\
\hline 9. & $\begin{array}{l}\text { Maniscalco, } \\
\text { et al., } 2021\end{array}$ & Italy & Cohort study & 95 patients & $\begin{array}{c}63 \\
\text { years old }\end{array}$ & $\begin{array}{l}6 \text { sessions per } \\
\text { week and }\end{array}$ & $\begin{array}{l}\text { Pulmonary rehabilitation consisted of: } \\
\text { 1. Physical exercise training (strengthen } \\
\text { groups of muscles in the upper and }\end{array}$ & $\begin{array}{l}\text { 1. PFT } \\
\text { 2. DLCO } \\
\text { 3. } 6 \mathrm{MWT}\end{array}$ & $\begin{array}{l}\text { Regardless of prior } \\
\text { cardiorespiratory } \\
\text { comorbidities, }\end{array}$ \\
\hline
\end{tabular}

seconds

Pulmonary

function

Lower limb muscle strength exercises specified in a three-tiered exercise plan with difficulty and inte

Pulmonary rehabilitation consisted of:

cardiopulmonary training (cycling,

3 weeks in post-COVID-

Strength exercise (weight training on resistance bands)

relaxation)

Nutritional

Occupational therapy and social

1. Anthropometric

monary rehabilitation Warm-up (5 minutes)

flexibility and mobility exercises

obic exercise set with walking

PFT and effective practice in the recovery of symptoms in well as an innovative strategy for the treatment of sequelae breathing (20 minutes) minutes) groups of muscles in the upper and 
$(80$ males,
15 females $)$

conducted for

5 weeks

lower extremities, treadmill walking,

and stationary cycling)

. Dietary counselling

3. Psychosocial counselling

10. Zampogna,

et al., 2020

Italy

Retrospective

data analysis

140 patients

71

1 session a day

with a

duration of 20

minutes per

day could be

increased to 2 -

3 sessions per

day for 30

minutes during

hospitalization

with an

average of 47

days

11. Liu, et al., China $\begin{aligned} & \text { Randomized } \\ & \text { controlled }\end{aligned}$
2020

study

72 patients

69

2 sessions

years old

(49 males,

23 females)

\section{every week fo}

6 weeks, once

a day for 10

minutes
Pulmonary rehabilitation consisted of:

1. Program level A: mobilization, active exercise and free walking, the activity of peripheral leg muscles, shoulders,

2. Program level B: gymnastics, strengthening, balance sports, and brisk walking. All exercises could be done without tools or using gymnastic equipment.

Interventions included:

1. Respiratory muscle exercise

2. Cough exercises

3. Diaphragm training

4. Stretching exercise

5. Gymnastics at home and full arms in circles multidisciplinary

rehabilitation may be

beneficial in post-COVID-19

patients in decreasing

sequelae following hospital

discharge.

Pulmonary rehabilitation in

post-COVID-19 patients

could significantly improve in standing balance, walking,

and standing tests from a

sitting position.

1.FEV1

2.FVC

3.DLCO

4.6MWT

5.QoL

6.ADL

7. Menta

rehabilitation can help elderly individuals with COVID-19

improve their respiratory

function, QoL, and decreasing anxiety, but it has little effect

on reducing depressive symptoms.

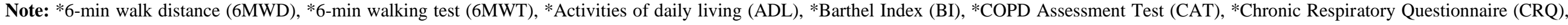

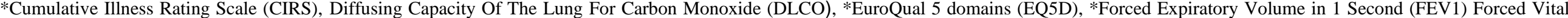

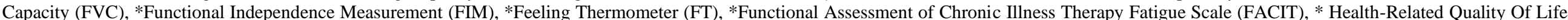

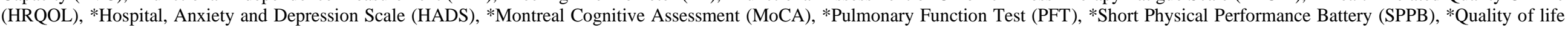
(QoL), 


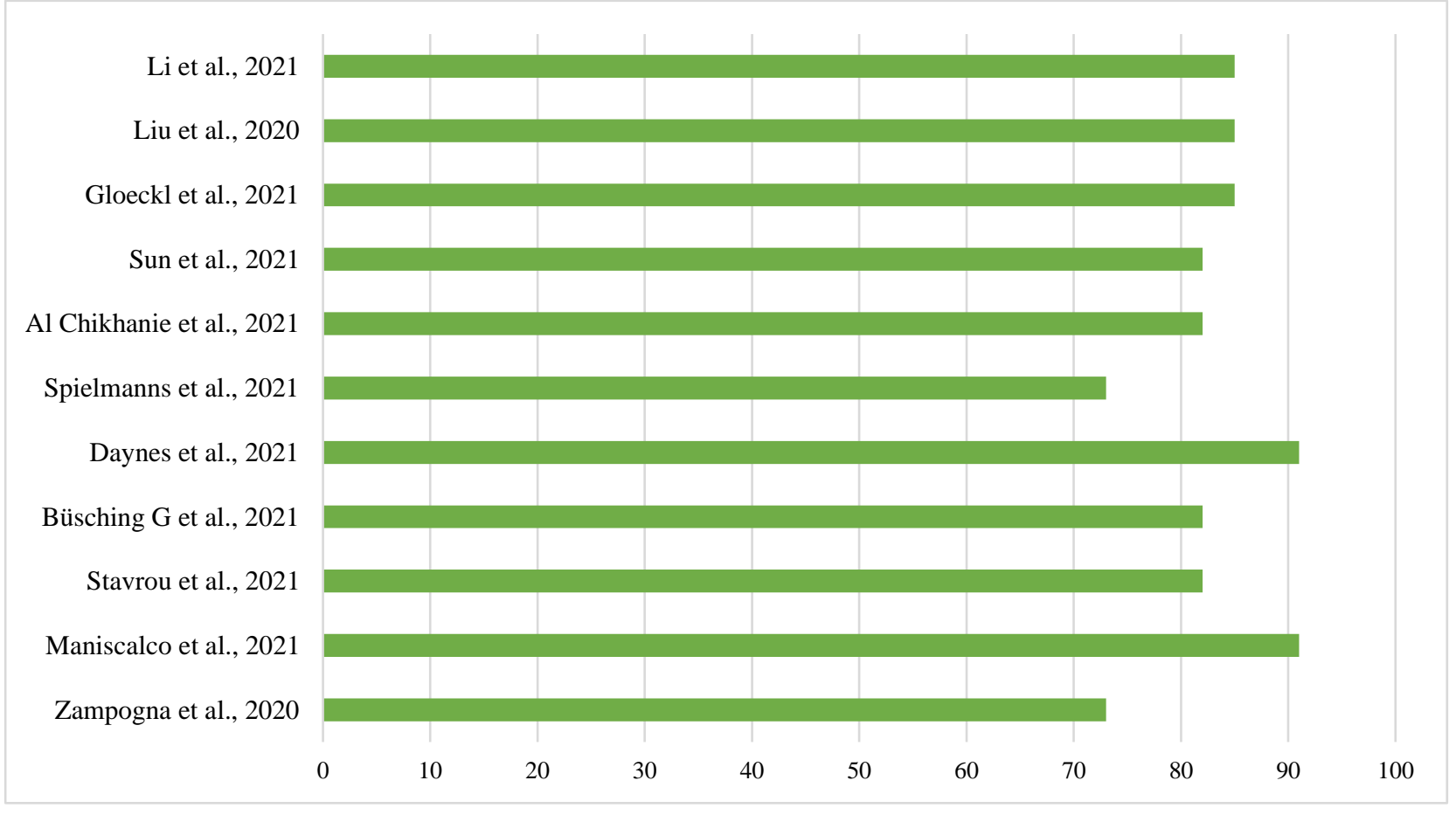

Figure 2. Risk of bias

\section{Intervention Characteristics}

Pulmonary rehabilitation provided to postCOVID-19 patients varied, including: aerobic $(n=8$ articles), ${ }^{10-17}$ breathing exercises (deep breathing, progressive muscle relaxation, breathing control, yoga, diaphragm breathing) $(\mathrm{n}=8$ articles $),{ }^{10,11,13,14,17-20}$ endurance training (lower limb muscle strength exercises, weight training on machines, free weight, elastic resistance bands, treadmill walking and stationary cycling) ( $\mathrm{n}=8$ articles), ${ }^{10-15,18,20}$ balance training ( $\mathrm{n}=3$ articles) ${ }^{10,16,18}$ relaxation $(\mathrm{n}=3$ articles $),{ }^{11,18,19}$ occupational therapy and psychological support $(\mathrm{n}=3$ articles), ${ }^{11,18,19}$ and nutrition consultation $(\mathrm{n}=2$ articles). ${ }^{18,20}$ Pulmonary rehabilitation could be provided directly by a psychotherapist $(\mathrm{n}=10 \text { articles })^{10-12,14-20}$ and could also be provided via tele-rehabilitation $(\mathrm{n}=1$ articles). ${ }^{13}$

The frequency of pulmonary rehabilitation varied from 2-6 sessions per week. Given for 1 session $(\mathrm{n}=2$ articles), ${ }^{10,16}$ given 2 sessions ( $\mathrm{n}=4$ articles), ${ }^{12,17-19}$ given 3 sessions $\left(\mathrm{n}=2\right.$ articles), ${ }^{13,14}$ given 4 sessions $(\mathrm{n}=1$ article),${ }^{11}$ given 6 sessions $(\mathrm{n}=1$ article $),{ }^{15}$ and not explained $\left(\mathrm{n}=1\right.$ article) ${ }^{20}$

The duration of pulmonary rehabilitation varied from 3-8 weeks, given for 3 weeks ( $n=4$ articles), ${ }^{11,18-20}$ administered for 5 weeks $\left(\mathrm{n}=1\right.$ article) ${ }^{15}$ administered for 6 weeks $\left(\mathrm{n}=4\right.$ articles), ${ }^{10,12,13,17}$ administered for 7 weeks $(\mathrm{n}=1 \text { article })^{16}$ and 8 weeks $\left(\mathrm{n}=1\right.$ article). ${ }^{14}$

Functional Independence Measure (FIM) parameters were used before starting pulmonary rehabilitation therapies in post-COVID-19 adult patients. ${ }^{11,20}$ Previously, post-COVID-19 adult patients had their daily activities monitored using activities of daily living (ADL) parameters to determine the results of the programmed pulmonary rehabilitation. ${ }^{17,19}$

Lung function was one of the parameters assessed following the implementation of pulmonary rehabilitation in COVID-19 survivor adult patients. Several parameters were used to assess lung function, including pulmonary function using spriometry (forced vital capacity (FVC), forced expiratory volume in 1 second (FEV1)) $\left(\mathrm{n}=6\right.$ article), ${ }^{10,11,13-15,17}$ bodyplethysmography $\left(\mathrm{n}=2\right.$ article), ${ }^{11,18}$ total lung capacity $\left(\mathrm{n}=1\right.$ article),${ }^{18}$ diffusing capacity of the lung for carbon monoxide (DLCO) ( $\mathrm{n}=4$ article), ${ }^{14,15,17,18}$ blood gas analysis $\left(\mathrm{n}=2\right.$ article), ${ }^{11,18} 6$-min walk test $(6 \mathrm{MWT})(\mathrm{n}=$ 8 article), ${ }^{10,11,13,15-18,20}$ endurance shuttle walk test (ESWT) $\left(\mathrm{n}=2\right.$ article), ${ }^{12,18}$ and modified medical research council dyspnea scale $(\mathrm{mMRC}) \quad(\mathrm{n}=2$ article). ${ }^{13,19}$

Pulmonary rehabilitation can help COVID-19 adult patients in managing stress as well as enhancing lung function. Several parameters were used to assess stress, including Hospital Anxiety and Depression Scale (HADS) $(\mathrm{n}=3 \text { article })^{10-12}$ and SAS anxiety and SDS depression scores $\left(\mathrm{n}=1\right.$ article). ${ }^{17}$

\section{DISCUSSION}

COVID-19 can disrupt respiratory, physical, and psychological functioning in hospitalized and COVID19 patients who just left the hospital. Patients with 
COVID-19-related complications need to require pulmonary rehabilitation to improve their physical and mental health andenable them to return to their families and society more quickly. ${ }^{21}$ Most non-critical COVID-19 pneumonia survivors have diminished diffusion capacity and Health-Related Quality of Life (HRQoL). Patients with COVID-19 pneumonia should have a pulmonary function test (PFT) and assesstheir QoL. ${ }^{22}$

Pulmonary rehabilitation is an individual rehabilitation treatment performed by health workers to patients with chronic lung disease after undergoing standard medical therapy. Pulmonary rehabilitation consists of comprehensive interventions, including psychological and nutritional support, andeducation and behaviour change with exercise training as the core. ${ }^{23}$ The purpose of pulmonary rehabilitation is to enhance physical ability, reduce psychological stress, and enhance QoL of COVID-19 survivors. ${ }^{24}$

The 6-minute walk test (6MWT) is commonly employed as an outcome metric in pulmonary rehabilitation programs. 6MWT is advised as an initial assessment for determining an appropriate measure of the effect of pulmonary rehabilitation. Effective 6MWT test was used to assess peak heart rate and oxygen desaturation in 70 individuals with Interstitial Pulmonary Fibrosis (IPF) who had undergone pulmonary rehabilitation. ${ }^{25,26}$

Every rehabilitation program should consider comorbidities that may influence a patient's progress or capacity to participate in the rehabilitation program. Any good rehabilitation program must include education about COVID-19. Since COVID-19 is a novel condition, patients must be educated about the disease implications and the potential consequences, such as post-COVID-19 sequelae following release from the hospital. ${ }^{24}$

Cardiovascular and pulmonary rehabilitation programs should include three main components: warming up, cooling down, and stretching. ${ }^{27}$ Pulmonary rehabilitation in COVID-19 survivors requires nutritional management, airway management, breathing exercises, physical activity, stretching, and manual therapy. ${ }^{28}$

Consensus statements of Taiwan Academy categorized patients with COVID-19 into five groups before undertaking cardiovascular and pulmonary rehabilitation programs. This is due to the rehabilitation program that will be given to post-COVID-19 patients is adjusted to the clinical condition of the patient and the patient's ability, hence it is safer and its effectiveness can provide optimal results. The five groups were: (1) patients with mild to moderate illness who have no risk factors, (2) patients with mild disease who have epidemiological risk factors, (3) patients with mild to moderate disease who have no risk factors, (4) ventilator-supported patients with no cognitive impairment, and (5) ventilator-supported patients with cognitive impairment. ${ }^{27}$

Rehabilitation programs that can be provided to post-COVID-19 patients include education regarding pulmonary rehabilitation, aerobic exercise, intermittent exercise, strength training, balance training, breathing exercises, and ADL improvement programs. ${ }^{7}$ Pulmonary rehabilitation education programs can be through booklets, videos, tele-health, andeducation provided to patients, including the importance of respiratory rehabilitation programs and healthy lifestyle education. $^{26,29}$ The recommended aerobic exercise is (walking, brisk walking, jogging, gymnastics, and swimming) an aerobic exercise program starting with low intensity for a short duration, around 3-5 sessions/week, with each session lasting between 20-30 minutes. $^{7,29}$ Endurance training is started with light weights and repeated 8-12 times, 1-3 sets/time, with a 2minute rest between sets, 2-3 sessions/week for six weeks. ${ }^{26,29}$ Breathing exercises include deep breathing, progressive muscle relaxation, breathing control, yoga, diaphragm breathing. ${ }^{7}$

Pulmonary rehabilitation programs for postCOVID-19 patients can be performed directly by psychotherapists or indirectly through tele-rehabilitation programs. Tele-rehabilitation can provide COVID-19 patients with psychological support and dietary counseling as part of their pulmonary rehabilitation. However, those who were unable to participate in a telerehabilitation program and had balance deficits should get treatment from a psychotherapist in person. COVID19 patients after being discharged from a hospitalsupervised multidisciplinary tele-rehabilitation program required discontinuation of the rehabilitation program at home. ${ }^{30}$

FIM tools assess post-COVID-19 patients before startingtherapy. It is used to determine the level of support required by individuals, ranging from those who need only minimal assistance to those who need total aid. The data obtained by FIM instruments can be used to track changes in impairment severity and examine rehabilitation outcomes as they occur throughout rehabilitation. According to the findings in FIM, comprehensive pulmonary rehabilitation after COVID19 is very beneficial. ${ }^{11,20}$

Regardless of the presence of pre-existing cardiorespiratory comorbidities, the rehabilitation intervention revealed statistically significant improvements in respiratory function, blood gases, and 
exercise ability in 95 post-COVID-19 patients. $^{15}$ Pulmonary rehabilitation significantly improved lung function and QoL in 36 post-COVID-19 adult patients after being discharged from the hospital compared to 36 patients who did not get pulmonary rehabilitation. ${ }^{17}$ In COVID-19 patients, pulmonary rehabilitation is a feasible, safe, and effective treatment, regardless of disease severity. Pulmonary rehabilitation program 2-5 sessions per day for three weeks significantly improved lung function and QoL in 50 patients with mild until severe COVID-19. ${ }^{18}$

In addition to improving lung function, pulmonary rehabilitation can help the patient to manage stress albeit not significant. Although considerable physical and psychosocial problems of post pulmonary rehabilitation remained in 21 COVID-19 post-ICU patients, pulmonary rehabilitation resulted in significant functional improvements in COVID-19 patients. $^{10}$ Pulmonary rehabilitation therapies were given to 30 adult COVID patients. The anxiety and depression levels on HADS improved, although the difference was not statistically significant. $^{12} 6$ weeks of respiratory rehabilitation reduced anxiety in older COVID-19 patients but did not significantlyto reduce depression. ${ }^{17}$

Since the average age of post-COVID-19 patients found in this study was 38-71 years old, a comprehensive evaluation of the patient's systemic function, particularly in terms of cognitive status, respiratory function, cardiovascular function, and musculoskeletal function, was required beforeconducting rehabilitation interventions in seriously ill and critically ill patients due to COVID-19 infection. Patients who were eligible for respiratory rehabilitation could receive pulmonary rehabilitation therapies. Before starting rehabilitation, approval from health workers was acquired and proper preparations were made in order to maximizing the patient's outcomes and minimizing unwanted risks. ${ }^{25,26}$

\section{CONCLUSION}

Most of the post-COVID-19 patients who returned from the hospital had residual symptoms in the form of cough, shortness of breath, and fatigue when performing activities, hence reducing their QoL. Pulmonary rehabilitation program is a comprehensive intervention that can be applied to reduce the sequelae felt by patients. The intervention must be guided by professional health workers, hence the benefits can be felt by the patient and to avoid complications. Methods of pulmonary rehabilitation can be either face-to-face with therapy or through tele-rehabilitation. This study proves that a comprehensive pulmonary rehabilitation program is effective in improving lung function in postCOVID-19 adult patients.

\section{Clinical Implication}

Scientifically reviewed studies have shown that providing interventions in the form of pulmonary rehabilitation effectively improves lung function in postCOVID-19 adult patients. The results of this study are certainly very useful in the field of medical science because they can help health workers provide interventions for post-COVID-19 patients. Face-to-face pulmonary rehabilitation can be an effective strategy in enhancing the intensity of lung function in patients. Further studies are needed to apply Tele-health-based pulmonary rehabilitation interviews guided by therapists or medical professionals through specific applications, hence they can be performed by post-COVID-19 patients at home.

\section{Acknowledgments}

We would like to extend our sincere thanks to the Head of Master Program in Nursing, Universitas Airlangga, and the Dean of the Faculty of Nursing, Universitas Airlangga for the supports in the preparation of this study.

\section{Confict of Interest}

The author stated there is no conflict of interest in this study.

\section{Funding}

This study does not receive any funding.

\section{Authors' Contributions}

Conceiving the study: H, TS, M, ANR. Designing the experiments: H. Gathering, analyzing, interpreting data, making tables and figures, writing the manuscript: $\mathrm{H}$, NCTR, HNA. Reviewing: TS, M, ANR. All authors contributed to revise the manuscript and approved the final version.

\section{REFERENCES}

1. Harapan H, Itoh N, Yufika A, Winardi W, Keam S, Te H. Coronavirus Disease 2019 (COVID-19): A Literature Review. J Infect Public Health. 2020;13:667-673. [PubMed]

2. WHO. WHO Coronavirus Disease (COVID-19) Dashboard [Serial Online]. 2021. [Cited 2021 September 29]. Available from: https://covid19.who.int/ 
3. Raman B, Cassar MP, Tunnicliffe EM, Filippini N, Griffanti L, Alfaro-Almagro F. Medium-Term Effects of SARS-CoV-2 Infection on Multiple Vital Organs, Exercise Capacity, Cognition, Quality of Life and Mental Health, Post-Hospital Discharge. EClinicalMedicine. 2021;31:100683. [PubMed]

4. Parry AH, Wani AH, Shah NN, Jehangir $M$. Medium-Term Chest Computed Tomography (CT) Follow-Up of COVID-19 Pneumonia Patients after Recovery to Assess the Rate of Resolution and Determine the Potential Predictors of Persistent Lung Changes. Egypt J Radiol Nucl Med. 2021;52(1). [CrossRef]

5. Sonnweber T, Sahanic S, Pizzini A, Luger A, Schwabl C, Sonnweber B. Cardiopulmonary Recovery after COVID-19: An Observational Prospective Multicentre Trial. Eur Respir J. 2021;57(4):2003481. [PubMed]

6. Huang C, Huang L, Wang Y, Li X, Ren L, Gu X. 6Month Consequences of COVID-19 in Patients Discharged from Hospital: A Cohort Study. Lancet. 2021;397(10270):220-32. [CrossRef] [PubMed]

7. Siddiq MAB, Rathore FA, Clegg D, Rasker JJ. Pulmonary Rehabilitation in COVID-19 Patients: A Scoping Review of Current Practice and Its Application during the Pandemic. Turkish J Phys Med Rehabil. 2021;66(4):480-94. [PubMed]

8. Page MJ, McKenzie JE, Bossuyt PM, Boutron I, Hoffmann TC, Mulrow CD. The PRISMA 2020 Statement: An Updated Guideline for Reporting Systematic Reviews. BMJ. 2021;372. [PubMed]

9. Moola S, Munn Z, Tufanaru C, Aromataris E, Sears K, Sfetcu R. JBI Manual for Evidence Synthesis [Serial Online]. 2020. [Cited 2021 September 29]. Available from: https://synthesismanual.jbi.global

10. Al Chikhanie Y, Veale D, Schoeffler M, Pépin JL, Verges S, Hérengt F. Effectiveness of Pulmonary Rehabilitation in COVID-19 Respiratory Failure Patients Post-ICU. Respir Physiol Neurobiol. 2021;287:103639. [PubMed]

11. Spielmanns M, Pekacka-Egli AM, Schoendorf S, Windisch W, Hermann M. Effects of a Comprehensive Pulmonary Rehabilitation in Severe Post-Covid-19 Patients. Int J Environ Res Public Health. 2021;18:2695. [PubMed]

12. Daynes E, Gerlis C, Chaplin E, Gardiner N, Singh SJ. Early Experiences of Rehabilitation for Individuals Post-COVID to Improve Fatigue, Breathlessness Exercise Capacity and Cognition A Cohort Study. Chron Respir Dis. 2021;18:1-4. [PubMed]

13. Li J, Xia W, Zhan C, Liu S, Yin Z, Wang J, et al. A Telerehabilitation Programme in Post-Discharge COVID-19 Patients (TERECO): A Randomised Controlled Trial. Thorax. 2021;0:1-10. [CrossRef]

14. Stavrou VT, Tourlakopoulos KN, Vavougios GD, Papayianni E, Kiribesi K, Maggoutas S. Eight Weeks Unsupervised Pulmonary Rehabilitation in Previously Hospitalized of SARS-CoV-2 Infection. J Pers Med. 2021;11(8):806. [CrossRef] [PubMed]

15. Maniscalco M, Fuschillo S, Ambrosino P, Martucci M. Preexisting Cardiorespiratory Comorbidity does not Preclude the Success of Multidisciplinary Rehabilitation in Post-COVID-19 Patients. Respir Med. 2021;184:106470. [PubMed]

16. Zampogna E, Zappa M, Spanevello A, Visca D. Pulmonary Rehabilitation and Asthma. Front Pharmacol. 2020;11:1-6. [CrossRef] [PubMed]

17. Liu K, Zhang W, Yang Y, Zhang J, Li Y, Chen Y. Respiratory Rehabilitation in Elderly Patients with COVID-19: A Randomized Controlled Study. Complement Ther Clin Pract. 2020;39:101166. [PubMed]

18. Gloeckl R, Leitl D, Jarosch I, Schneeberger T, Nell C, Stenzel N. Benefits of Pulmonary Rehabilitation in COVID-19: A Prospective Observational Cohort Study. ERJ Open Res. 2021;7:00108-2021. [CrossRef] [PubMed]

19. Sun J, Liu J, Li H, Shang C, Li T, Ji W. Pulmonary Rehabilitation Focusing on the Regulation of Respiratory Movement Can Improve Prognosis of Severe Patients with COVID-19. Ann Palliat Med. 2021;10(4):4262-4272. [CrossRef] [PubMed]

20. Büsching G, Zhang Z, Schmid JP, Sigrist T, Khatami R. Effectiveness of Pulmonary Rehabilitation in Severe and Critically Ill COVID19 Patients: A Controlled Study. Int J Enviromental Res Public Heal. 2021;18:8956. [CrossRef] [PubMed]

21. Yang L-L, Yang T. Pulmonary Rehabilitation for Patients with Coronavirus Disease 2019 (COVID19). Chronic Dis Transl Med. 2020;6(2):79-86. [PubMed]

22. van der Sar - van der Brugge S, Talman S, Boonman - de Winter LJM, de Mol M, Hoefman E, van Etten RW. Pulmonary Function and Health-Related Quality of Life after COVID-19 Pneumonia. Respir Med. 2021;176:106272. [PubMed]

23. Yang L-L, Yang T. Pulmonary Rehabilitation for Patients with Coronavirus Disease 2019 (COVID19). Chronic Dis Transl Med. 2020;6(2):79-86. [PubMed]

24. Barker-Davies RM, O'Sullivan O, Senaratne KPP, Baker P, Cranley M, Dharm-Datta S. The Stanford Hall Consensus Statement for Post-COVID-19 Rehabilitation. Br J Sports Med. 2020;54(16):949_ 959. [CrossRef]

25. Holland AE, Hill CJ, Dowman L, Glaspole I, Goh N, Lee AL. Short-and Long-Term Reliability of the 6-Minute Walk Test in People with Idiopathic Pulmonary Fibrosis. Respir Care. 2018;63(8):9941001. [CrossRef] [PubMed]

26. Zhao HM, Xie YX, Wang C. Recommendations for Respiratory Rehabilitation in Adults with Coronavirus Disease 2019. Chin Med J (Engl). 2020;133(13):1595-1602. [CrossRef] [PubMed]

27. Cheng YY, Chen CM, Huang WC, Chiang SL, Hsieh PC, Lin KL. Rehabilitation Programs for Patients with CoronaVirus Disease 2019: Consensus Statements of Taiwan Academy of Cardiovascular and Pulmonary Rehabilitation. J Formos Med Assoc. 2021;120(1):83-92. [PubMed]

28. Wang TJ, Chau B, Lui M, Lam GT, Lin N, Humbert S. Physical Medicine and Rehabilitation and 
Pulmonary Rehabilitation for COVID-19. Am J Phys Med Rehabil. 2020;99(9):769-74. [CrossRef] [PubMed]

29. Demeco A, Marotta N, Barletta M, Pino I, Marinaro C, Petraroli A. Rehabilitation of Patients PostCOVID-19 Infection: A Literature Review. J Int Med Res. 2020;48(8):1-10 [CrossRef] [PubMed]
30. Salawu A, Green A, Crooks MG, Brixey N, Ross DH, Sivan M. A Proposal for Multidisciplinary Tele-Rehabilitation in the Assessment and Rehabilitation of COVID-19 Survivors. Int J Environ Res Public Health. 2020;17(13):1-13. [CrossRef] [PubMed] 